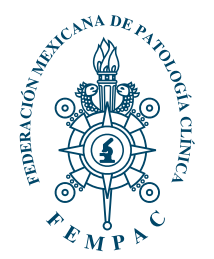

\title{
HbA1c: aplicando objetivos de calidad IFCC en laboratorios mexicanos
}

\author{
HbA1c: Applying IFCC quality targets to Mexican laboratories
}

\author{
Rojano-Rodríguez Eduardo,* Sierra-Amor Rosa Isabel ${ }^{\ddagger}$
}

Palabras clave: HbA1c, IFCC, objetivos de calidad, Six Sigma Metrics, evaluación de métodos.

Keywords:

HbA1C, IFCC, quality targets, Six Sigma Metrics, methods evaluation.

* Coordinador del Programa Educacional del Laboratorio Europeo de Referencia para Glicohemoglobina, Grupo México.

Coordinador del

Proyecto EurA1c del

Comité de Educación en el Uso de Biomarcadores de Diabetes. Laboratorios Biomédicos Pánuco.

Veracruz, México.

${ }^{\ddagger}$ Laboratorio Laquims,

S.C. Representante

regional de la

IFCC. México.

Correspondencia:

MAC Eduardo

Rojano-Rodríguez Privada Abasolo S/N Col. Caballero, 93990, Pánuco, Ver., México. E-mail: edrojano@ prodigy.net.mx

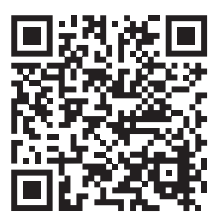

RESUMEN

La Federación Internacional de Química Clínica y Medicina de Laboratorio (IFCC) ha realizado durante décadas grandes esfuerzos para lograr la estandarización de la prueba de HbA1c, además ha desarrollado un modelo de objetivos de calidad establecido mediante una fuerza de tarea, que propuso un criterio inicial: un error máximo permisible de 5 $\mathrm{mmol} / \mathrm{mol}(0.46 \%)$ en un nivel de dos sigmas. Laboratorios mexicanos han participado en programas organizados por la IFCC, en los cuales se utilizan muestras con un valor asignado por el método de referencia internacional como lo es el proyecto denominado EurA1c del Comité de Educación en el Uso de Biomarcadores en Diabetes, que incluye la participación de más de 4,000 laboratorios. En el marco de este modelo, se realizó una evaluación del desempeño de los laboratorios mexicanos participantes para conocer el estado de calidad y compararlo con el de otros países. En los resultados se observó una alta variabilidad en los desempeños, siendo la principal fuente de error una relativa alta imprecisión. Sin embargo, pudo detectarse también una tendencia de mejora. A través de la educación y una participación organizada se puede lograr que se cumpla con el objetivo de calidad propuesto.

\section{ABSTRACT}

For decades, the International Federation of Clinical Chemistry and Laboratory Medicine (IFCC) has made great efforts and achievements in standardization of $\mathrm{HbA1c}$ testing. A model was developed for quality objectives for $\mathrm{HbA1C}$, the IFCC task force-HbA1c proposed an initial criteria of a total allowable error of $5 \mathrm{mmol} / \mathrm{mol}(0.46 \%)$ at a level of two sigma. Mexican laboratories have participated in projects organized by the IFCC using samples with a target value determined by the international reference method, such as the project EurA1c of the Committee on Education in the Use of Biomarkers in Diabetes, which includes the participation of 4,000 laboratories. An evaluation of the performance of the participating Mexicans laboratories was carried out within the framework of this model to know the quality status and compared with other countries. In the results, a high variability in performance was observed, the main source of error being a relatively high imprecision. However, an improvement trend was also detected. The proposed quality objective could be achieved through education and organized participation.

\section{INTRODUCCIÓN}

U na vez conocida la utilidad clínica de las determinaciones de la hemoglobina glicosilada (HbA1c) en el control de las complicaciones de los pacientes con diabetes, ${ }^{1,2}$ y debido una falta de estandarización de estas determinaciones, la Federación Internacional de Química Clínica (IFCC) formó un grupo de trabajo en HbA1c que desarrolló un sistema internacional para la estandarización de esta prueba, ${ }^{3}$ se realizaron diversas actividades para este fin, como la elaboración de un calibrador primario, ${ }^{4,5}$ el desarrollo de un método de referencia internacional (RMP), ${ }^{6}$ además de colaboraciones con las principales instituciones internacionales en diabetes y la industria. ${ }^{7}$ Gracias a estas acciones, se consiguió el establecimiento de un sistema internacional para la estandarización de las determinaciones de $\mathrm{HbA1c}$, el cual es actualmente reconocido como la única ancla válida para este fin, se encuentra vigente y operando una red de laboratorios de referencia a nivel internacional,

Citar como: Rojano-Rodríguez E, Sierra-Amor RI. HbA1c: aplicando objetivos de calidad IFCC en laboratorios mexicanos. Rev Mex Patol Clin Med Lab. 2020; 67 (4): 190-197. https://dx.doi.org/10.35366/99466 
lo cual ha permitido a los fabricantes estandarizar sus metodologías al RMP en el ámbito mundial. ${ }^{8}$

Después, la IFCC puso en marcha una fuerza de tarea en la implementación de la estandarización de $\mathrm{HbA1c}$ (TF-HbA1c) ${ }^{9}$ encaminada principalmente al desarrollo de objetivos de calidad para las mediciones de HbA1c. Tomando en cuenta estos objetivos de calidad como base, y en conjunto con grupos profesionales, se proponía asesorar en el uso de estas determinaciones en el monitoreo y el diagnóstico de diabetes e intolerancia a la glucosa. La TF-HbA1c propuso un modelo para establecer y desarrollar estos objetivos de calidad, el cual es adecuado para estimar el estatus de calidad a diferentes niveles, por ejemplo, en el ámbito individual (intralaboratorio), fabricante, país (interlaboratorios) y entre países. ${ }^{10}$

Establecida la estandarización mundial y propuesto este modelo, la IFCC priorizó la educación en diabetes, para ello instituyó un nuevo comité enfocado en aspectos relacionados con el monitoreo y diagnóstico de la diabetes, así como la evaluación de nuevos marcadores útiles para estos propósitos. Este comité, denominado Comité Para la Educación en el Uso de Biomarcadores en Diabetes (C-EUBD), ${ }^{11}$ está formado por un grupo de notables científicos y expertos en el tema. A través de éste se implementó el proyecto EurA1c, mismo que ha tenido gran éxito y aceptación con amplía colaboración mundial y ha trascendido a otros continentes como América, Asia y África.

En México, a través del tiempo hemos colaborado con estos esfuerzos a través de la realización de diversas actividades, entre ellas la participación de un grupo de laboratorios mexicanos en el Programa Educacional del Laboratorio Europeo de Referencia para Glicohemoglobina $^{12}$ y, desde 2017, un grupo creciente de laboratorios ha participado en el proyecto EurA1c de la IFCC a través del C-EUBD. ${ }^{13}$

El modelo para objetivos de calidad de la TF-HbA1c fue aplicado para evaluar el estatus de calidad en diferentes niveles de los laboratorios mexicanos participantes en estos programas, los resultados muestran variabilidad en el desempeño de los participantes con el mayor aporte proveniente de una relativa alta imprecisión, también se observó que la participación continua en estos programas ha llevado a una mejora del desempeño promedio hacia al objetivo de calidad establecido en todos los niveles.

\section{MATERIAL Y MÉTODOS}

Durante 2019 se realizó una invitación a laboratorios mexicanos para participar en el ensayo EurA1c 2019 auspiciado por el C-EUBD de la IFCC. Después de realizar una amplia difusión por diversos medios, se logró la conformación de un grupo que se denominó ERL-México, el cual contó con 47 laboratorios de distintos niveles de complejidad representantes de 15 estados de la República Mexicana.

Se utilizó el modelo para objetivos de calidad propuesto por la TF-HbA1c de la IFCC para estimar el desempeño de los laboratorios participantes en el ámbito intralaboratorio, por país (interlaboratorios) e internacional, se usaron los datos obtenidos de los programas en que participan este grupo de laboratorios mexicanos, ambos programas emplean muestras con un valor asignado utilizando el RMP. En el programa educacional del Laboratorio Europeo de Referencia en Glicohemoglobina (ERL), se utilizan muestras con valor asignado por este mismo laboratorio de referencia, en tanto que en el proyecto EurA1c se utilizan muestras con valor asignado por cinco laboratorios de referencia de la red de laboratorios certificados de la IFCC.

Para el nivel intralaboratorios se utilizaron los resultados del ejercicio 2019 del programa educacional ERL, para el análisis entrelaboratorios los resultados de un grupo de 47 participantes en el proyecto EurA1c 2019, en tanto que para la comparación entre países se utilizan los resultados acumulados del EurA1c-2019, incluyendo a México, a manera de perspectiva internacional.

El modelo propuesto por la TF-HbA1c está basado en el sistema Sigma-Metrics (SM), una estrategia para la administración de la calidad que ofrece un parámetro universal para evaluar el desempeño de un proceso y puede categorizarlo mediante un valor sigma en un rango que varía entre dos y seis, entre mayor sea el valor sigma $(\sigma)$ menor es el riesgo de fallar el criterio, lo que hace al proceso más confiable. ${ }^{14}$

El modelo incluye características analíticas como la imprecisión y la veracidad dentro de un marco de especificaciones clínicas concretas, delimitadas por un error total permisible (TEA, por sus siglas en inglés). Tanto el TEA como el nivel de riesgo pueden ser elegidos libremente haciendo un modelo más flexible en contraste con el modelo basado en variabilidad biológica (VB), en donde los límites de desempeño son más estrechos y varían de acuerdo con la concentración de HbA1c, por lo que, para la evaluación realizada por la TF-HbA1c de estos dos principios se eligió una concentración de HbA1c de $50 \mathrm{mmol} / \mathrm{mol}$. Se conservaron los criterios de VB para denotar los más estrictos niveles de desempeño en tres categorías: mínimo, aceptable y óptimo en la representación gráfica del modelo. ${ }^{10}$

La TF-HbA1c ha establecido como punto de partida un criterio mínimo de desempeño para los laboratorios 
de rutina ejecutando determinaciones de $\mathrm{HbA} 1 \mathrm{c}$, el cual consiste en un TAE de $5 \mathrm{mmol} / \mathrm{mol}(10 \%$ a $50 \mathrm{mmol} / \mathrm{mol}$ ) y un valor sigma mínimo de dos $(\sigma=2)$, mientras que para los laboratorios que realicen ensayos clínicos se recomienda el mismo TAE con un valor sigma de cuatro $(\sigma=4) .{ }^{10}$

El valor de TAE fue seleccionado de acuerdo con el primer nivel de la jerarquía del consenso de Estocolmo. ${ }^{15}$ Los $5 \mathrm{mmol} / \mathrm{mol}$ están basados en la diferencia de dos determinaciones consecutivas de $\mathrm{HbA} 1 \mathrm{c}$ que indican al personal médico cambiar la terapia establecida, es también la diferencia entre el nivel superior de individuos en riesgo y el criterio por el cual se establece el diagnóstico de diabetes. ${ }^{16}$

El desempeño de un laboratorio expresado en un valor sigma $(\sigma)$ es calculado de acuerdo con la siguiente ecuación:

$$
\sigma=\frac{(\mathrm{TAE}-|\mathrm{B}|)}{\mathrm{I}}
$$

Siendo $\sigma$ el riesgo de fallar el criterio, TAE el error total permisible, $|\mathrm{B}|$ es el sesgo en unidades absolutas, I es la imprecisión en unidades absolutas.

Asimismo, y de acuerdo con el consenso global sobre el reporte de valores de $\mathrm{HbA} 1 \mathrm{c}$, se reportan los resultados en ambas concentraciones, IFCC y NGSP, siendo los factores de conversión los siguientes:

- A1c en unidades de concentración: NGSP = 0.09151 IFCC +2.15

- Sesgo absoluto en unidades de concentración:

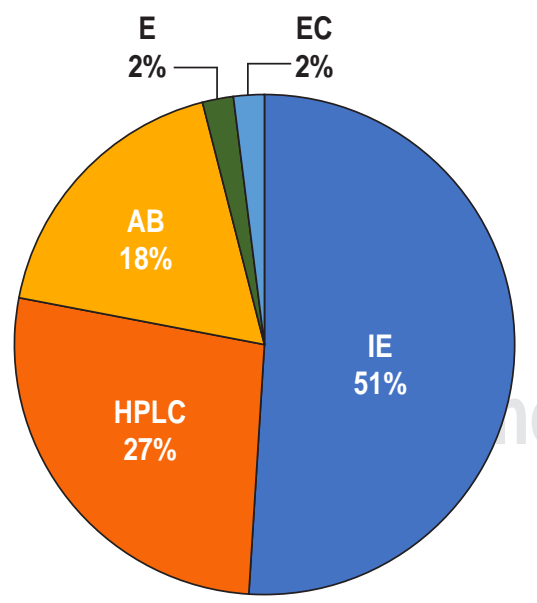

Figura 1: Distribución de laboratorios participantes por metodología. $\mathrm{HPLC}=$ cromatografía de líquidos de alto desempeño, IE = inmunoensayo, $\mathrm{AB}=$ afinidad al boronato $\mathrm{E}=$ enzimático, $\mathrm{EC}=$ electroforesis capilar.

\begin{tabular}{lc}
\multicolumn{1}{c}{$\begin{array}{c}\text { Tabla 1: Equipos utilizados por los } \\
\text { laboratorios participantes. }\end{array}$} & $\begin{array}{c}\text { No. de } \\
\text { laboratorios }\end{array}$ \\
\hline Equipo & 8 \\
\hline Bio-Rad D-10_A1C_Program & 6 \\
Abbott ARCHITECT Inmuno essay (LN 2K96) & 6 \\
Siemens DCA 2000/Nantage & 5 \\
NycoCard Reader II & 4 \\
Siemens Dimension RxL analyzer & 2 \\
Bio-Rad Variant-II_Turbo_A1C_Program & 2 \\
SPIN 200 E (SPINREACT) Inmuno Ensayo Turb... & 2 \\
LIFOTRONIC GH900 & 2 \\
Vitros 5.1 fusion Ortho diagnostics & 2 \\
Roche Diagnostics Tina-quant, Whole Blood, Gen.3 & 2 \\
Alere Afinion & 2 \\
Otros & 1 \\
SEBIA Capillarys 2 flex-piercing & 1 \\
Ceragem Labona Check A1c & 1 \\
Sekisui CS T240 & 1 \\
Taurus Twin IL Werfen & \\
\hline
\end{tabular}

NGSP $=0.09151$ IFCC

- Imprecisión en CV\% a $50 \mathrm{mmol} / \mathrm{mol}$ : NGSP $=0.6851$ IFCC

- Imprecisión en CV\% en unidades NGSP: IFCC $=1.46 \mathrm{NGSP}$

Para el análisis estadístico de los resultados de los participantes se utilizó la herramienta Microsoft ${ }^{\circledR}$ Excel 2016 (Microsoft Corporation) para calcular media, coeficiente de variación (CV\%), sesgo, desviación estándar (DE), error total (TE), error máximo permisible (TEA), regresión lineal y coeficiente de correlación.

\section{RESULTADOS}

De los participantes de 15 estados de la República, la CDMX, Veracruz y Yucatán registraron la mayor participación $(n=9)$, los participantes fueron $70 \%$ de laboratorios del sector privado y $30 \%$ del sector público.

Los métodos utilizados para realizar las determinaciones de $\mathrm{HbA} 1 \mathrm{c}$ por los participantes fueron principalmente basados en el principio de inmunoensayo $(n=23)$, seguido de cromatografía de líquidos de alto desempeño (HPLC) $(\mathrm{n}=12)$ (Figura 1).

Los equipos utilizados por los participantes se mencionan en la Tabla 1; el más utilizado en el grupo ERL-México fue el equipo D-10 de la marca Bio-Rad, seguido de los equipos DCA-Vantage de la marca Siemens y ARCHITECT de Abbott. 


\section{Nivel intralaboratorio}

Para estimar el desempeño intraindividual se aplicó el modelo propuesto por la TF-HbA1c de la IFCC a ocho participantes del programa ERL 2019 (Figura 2), observándose que tres cumplían el criterio mínimo de un TAE $=10 \%$ y un valor de dos sigma, de éstos, un participante utilizó un equipo basado en electroforesis capilar, alcanzando el nivel deseable en el modelo de VB, superando el nivel sigma de seis $(\sigma=6.6)$. En tanto otro que utilizó un método HPLC se ubicó por debajo del criterio siguiente inferior $(\sigma=3.1)$ y cinco más no logran el criterio mínimo. Asimismo, el desempeño global quedó fuera del área que significa cumplir el criterio establecido $(\sigma=0.85)$.

\section{Nivel interlaboratorios}

Se sometieron al modelo los resultados obtenidos por grupo ERL-México en el EurA1c 2019 (Figura 3), que incluyó un total de 27 pares de datos provenientes de los laboratorios participantes después de eliminar resul-

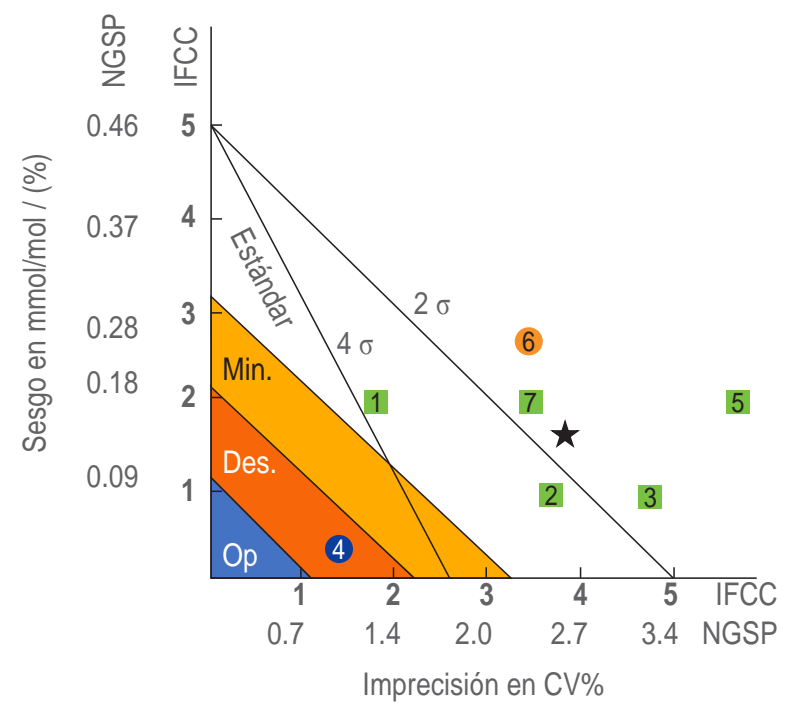

Figura 2: Modelo aplicado a ocho laboratorios del programa educacional anual del ERL 2019. Imprecisión como CV\% en el eje X; veracidad como sesgo absoluto a $50 \mathrm{mmol} / \mathrm{mol}$ en el eje $Y$. La estrella negra representa el promedio global de todos los participantes. Los números corresponden a los laboratorios participantes, los colores a los equipos empleados en la determinación: D-10 Bio-Rad (verde), DCA Vantage Siemens (naranja), Capillarys 2 flex-piercing Sebia (azul), Tina-quant Gen 3 Roche (lila). Los círculos y cuadros representan la desviación positiva o negativa respectivamente.

NGSP $=$ National Glycohemoglobin. Standardization Program; IFCC $=$ Federación Internacional de Química Clínica y Medicina de Laboratorio; CV\% = coeficiente de variación; Min. = Mínimo; Des. = Deseable; Op = Óptimo .

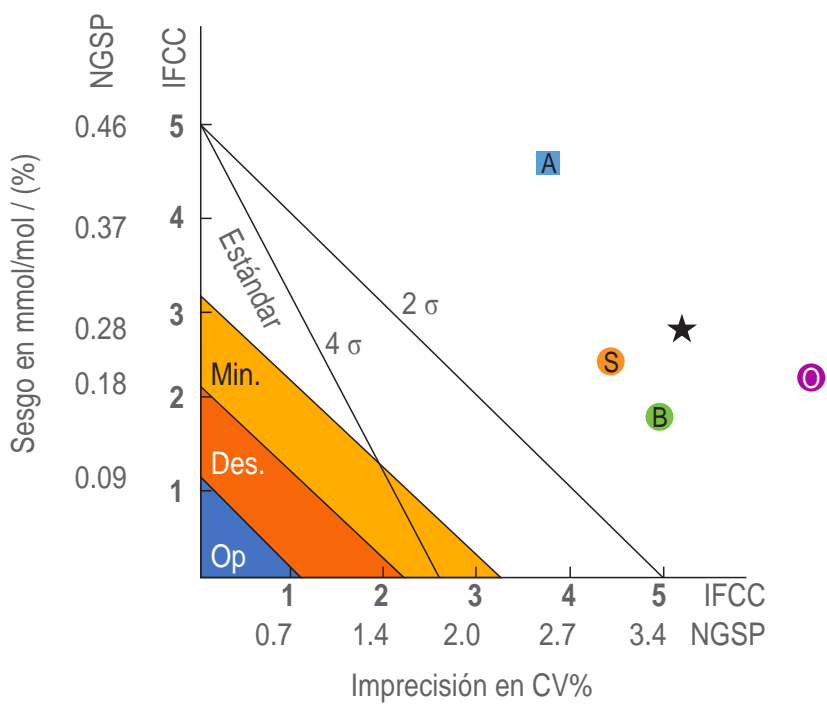

Figura 3: Modelo aplicado a resultados del grupo ERL-México en proyecto EurA1c 2019. Imprecisión como CV\% en el eje X; veracidad como sesgo absoluto a $50 \mathrm{mmol} / \mathrm{mol}$ en el eje Y. La estrella negra representa el resultado global de todos los participantes en los grupos analizados. Las letras corresponden a las metodologías y fabricantes Bio-Rad (B), inmunoensayo Siemens (S), inmunoensayo Abbott ARCHITECT (A) y otros (0). Los círculos y cuadros representan la desviación positiva o negativa respectivamente.

NGSP = National Glycohemoglobin. Standardization Program; IFCC $=$ Federación Internacional de Química Clínica y Medicina de Laboratorio; $\mathrm{CV} \%=$ coeficiente de variación; Min. = Mínimo; Des. = Deseable; Op = Óptimo.

tados atípicos que excedieron $25 \%$ del valor asignado a las muestras de acuerdo con el criterio establecido por el organizador del proyecto. Se agruparon por fabricante y metodología empleada en las determinaciones de HbA1c, logrando conformar los siguientes grupos $(n \geq 6)$ : participantes usando equipos HPLC del fabricante Bio-Rad ( $n$ $=9$ ), inmunoensayo de Siemens $(n=6)$, inmunoensayo ARCHITECT Abbott $(n=6)$, el resto de fabricantes con pocos equipos representados $(n<5)$, se clasificaron como otros $(n=22)$. Ninguno de los grupos formados logra el criterio mínimo, por lo tanto, el desempeño global también quedó fuera del área que significa cumplir el criterio establecido $(\sigma=0.5)$.

\section{Nivel entre países}

En la forma más global de aplicación del modelo se incluyeron resultados de algunos países que recibieron muestra liofilizada por parte del organizador del proyecto EurA1C 2019 (Figura 4). Estos países fueron Francia $(n=752)$, Grecia $(n=78)$, internacional (un grupo de países [ $n=43]$ ), México $(n=42)$, Portugal $(n=43)$, Tailandia $(n=185)$ 
y Turquía $(n=50)$. De estos países, solamente el grupo internacional logró el criterio mínimo, ninguno alcanzó el segundo criterio propuesto $(\sigma=4)$, ni el nivel mínimo en el modelo de VB. Seis países no lograron el criterio mínimo, incluyendo a México, en tanto que el desempeño global tampoco lo alcanzó, con un CV\% de $6.2 \%$, sesgo de $1.1 \mathrm{mmol} / \mathrm{mol}$ y una puntuación sigma $=0.9$.

\section{DISCUSIÓN}

Aplicamos el modelo descrito por la TF-HbA1c para obtener una comparación del desempeño de los participantes en los tres niveles, la cual facilita comprender las variables que afectan primordialmente a sus resultados en los dos programas internacionales, empleando muestras con un valor asignado por el MRP de la IFCC. En ambos casos, calculamos el desempeño promedio como una referencia que orienta sobre el estatus de cada laboratorio, grupo o país promoviendo su mejora, primeramente al desempeño promedio para unos y para otros la búsqueda de más estrictos objetivos de calidad que garanticen una alta confiabilidad en sus resultados.

En el nivel intralaboratorio, 38\% de los participantes cumplen el criterio mínimo establecido de TAE $=5 \mathrm{mmol} /$

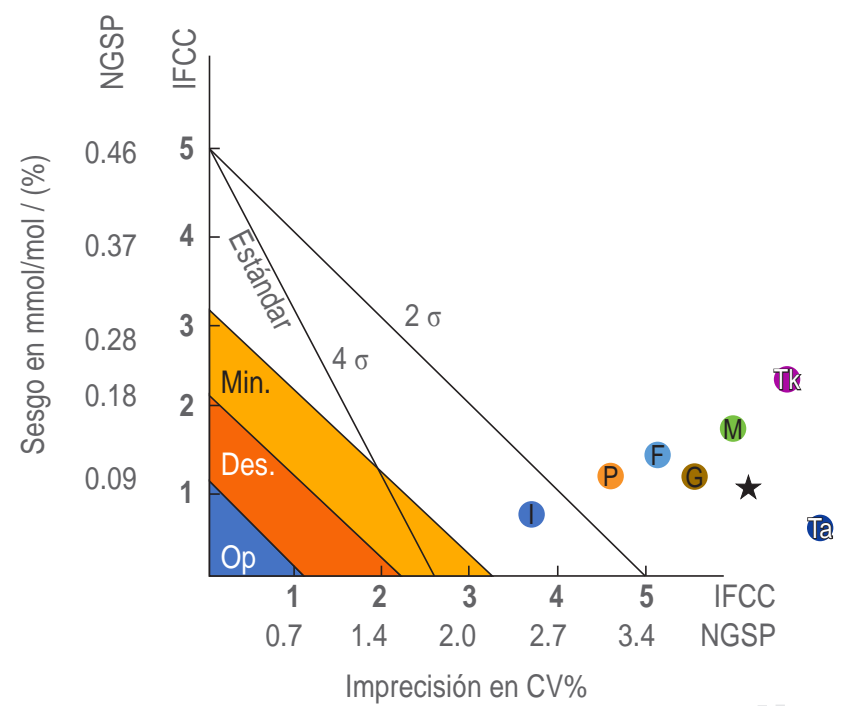

Figura 4: Modelo aplicado a países participantes en el proyecto EurA1c 2019. Imprecisión como CV\% en el eje X; veracidad como sesgo absoluto a $50 \mathrm{mmol} / \mathrm{mol}$ en el eje Y. La estrella negra representa el promedio global de los países en el EurA1c usando liofilizado $(n=$ 16). Las letras corresponden a países: Francia (F), Grecia (G), Internacional (I), México (M), Portugal (P), Tailandia (Ta) y Turquía (Tk). NGSP $=$ National Glycohemoglobin. Standardization Program; IFCC $=$ Federación Internacional de Química Clínica y Medicina de Laboratorio; CV\% = coeficiente de variación; Min. = Mínimo; Des. = Deseable; Op = Óptimo .

\begin{tabular}{lcc}
\multicolumn{3}{c}{$\begin{array}{c}\text { Tabla 2: Puntuación sigma por participante } \\
\text { por periodo de participación. }\end{array}$} \\
\cline { 2 - 3 } Instrumento & \multicolumn{2}{c}{ Puntuación $\sigma$} \\
\cline { 2 - 3 } & Periodo 1 & Periodo 2 \\
\hline D-10_A1c & 3.07 & 5.4 \\
D-10_A1c & 5.4 & 6.5 \\
Capillarys 2 flex-piercing & 1.66 & 1.73 \\
Vantage (DCA 2000) DCCT\% & 0.3 & 1.74 \\
\hline
\end{tabular}

mol y un valor de sigma de dos (Figura 2), destacando que un participante usando un equipo de electroforesis capilar mostró un desempeño considerado de clase mundial $(\sigma=$ 6.6), ubicándose en el nivel deseable del modelo basado en VB. Los otros participantes que cumplen el criterio emplearon un equipo de HPLC, observándose que todos los participantes que usaron este mismo equipo, independientemente de lograr el objetivo de calidad, mostraron una desviación negativa del valor asignado. El desempeño promedio fue próximo al límite mínimo aceptable; sin embargo, aunque no se superó este objetivo, se puede destacar una mejora constante de su desempeño en este programa educacional (Tabla 2).

Los resultados entre laboratorios muestran una más amplia dispersión con una relativa mayor desviación del valor asignado (Figura 3), se ven afectados por algunos factores como son una mayor heterogeneidad, ya que incluyen una gran diversidad de plataformas y metodologías desde dispositivos punto de atención (POCT) hasta equipos dedicados de alto desempeño, así como una relativa baja participación.

Aunque en el proyecto EurA1c está demostrada la conmutabilidad de las muestras y no se observa diferencia estadísticamente significativa entre los resultados de muestra fresca y liofilizada, también se hace referencia a que algunos dispositivos POCT no son adecuados para procesar las muestras liofilizadas, ${ }^{12}$ debido a que pueden verse afectados por un efecto de matriz de la muestra; esto también puede ser la razón por la que los resultados de un grupo de participantes constituido por analizadores NycoCard Reader II $(n=5)$ tuvieran que ser excluidos del análisis estadístico al mostrar una amplia desviación positiva que excedió $25 \%$ del valor asignado. En otro instrumento, basado en el mismo principio que el anterior, denominado Alere Afinion $(n=2)$, no se obtuvo ningún resultado al procesar la muestra. Este instrumento es ampliamente usado en Europa ( $n=269)$ y utilizando muestra fresca aprobó el objetivo de calidad propuesto, el primer instrumento mencionado no se incluyó en la lista 
de estos participantes, ${ }^{12}$ este tema deberá ser estudiado con mayor detalle.

La aplicación del modelo entre países muestra resultados más homogéneos con el mayor aporte al error total proveniente de la imprecisión. De los 25 grupos de datos, se reportó un sesgo medio de $0.4 \mathrm{mmol} / \mathrm{mol}$ y un $5.8 \mathrm{CV} \%$, se graficó este promedio y el de siete países de distintos niveles: uno dentro de rango aceptable, cuatro por debajo del promedio acumulado y próximos al criterio, así como dos por encima del promedio. En los resultados del EurA1c-2019 México dejó de ser el más alto $\mathrm{CV} \%$ obteniendo $(\mathrm{CV} \%=6.1)$, como se mencionó antes, también se observó una mejora constante hacia el objetivo de calidad señalado con cada participación (Tabla 3).

Países que han organizado programas de ensayos de aptitud para HbA1c por décadas han tenido mejoras paulatinas que son producto de una mayor estandarización o armonización de los resultados. ${ }^{17}$ Por ejemplo, el programa de ensayos de aptitud del College of American Pathologist (CAP) en los Estados Unidos ha ido reduciendo el criterio de aprobación de sus participantes que inició por encima de 10\% como CV y para el ejercicio 2019 $\mathrm{GH}-5$, el criterio de aprobación fue establecido en seis por ciento (6\% como CV\%). ${ }^{18}$

En el ensayo CAP 2019 GH5-A, dos muestras fueron procesadas por más de 3,100 participantes cada una, éstas tenían niveles de concentración similares a las usadas en la participación de los laboratorios mexicanos en el EurA1c-2019, GH5-01 = 5.46\% y GH5-03 $=9.31 \%$ (36.1 y $78.4 \mathrm{mmol} / \mathrm{mol}$ respectivamente), se observó un promedio de $\mathrm{CV} \%=3.4 \%$ y un sesgo medio $=0.015 \%$ (NGSP). ${ }^{19}$ Este desempeño se ubica cercano al límite, aunque fuera del área de aprobación en el modelo de la TF-HbA1c $(\sigma=1.0)$, se debe recordar que aun cuando el $\mathrm{CV} \%$ de estas muestras es numéricamente inferior al utilizar unidades NGSP (\%) los rangos son estrechados, ya que los valores son diferentes. ${ }^{20,21}$ Por otro lado, siete de los ocho laboratorios mexicanos en la Figura 2 mostraron

\begin{tabular}{|c|c|c|}
\hline \multicolumn{3}{|c|}{$\begin{array}{l}\text { Tabla 3: Variación de sesgo y CV\% por } \\
\text { periodo de participación grupo ERL-México } \\
\text { en el proyecto EurA1c del C-EUBD. }\end{array}$} \\
\hline Periodo & $\begin{array}{c}\text { Veracidad } \\
(\mathrm{mmol} / \mathrm{mol})\end{array}$ & $\begin{array}{l}\text { Imprecisión } \\
\text { (CV\%) }\end{array}$ \\
\hline EurA1c-2017 & -0.3 & 12.7 \\
\hline EurA1c-2018 & -0.4 & 9.6 \\
\hline EurA1c-2019 & 1.72 & 6.1 \\
\hline
\end{tabular}

un promedio menor a este criterio de aceptación (6 CV\%) en las dos muestras utilizadas.

Las evaluaciones a través del tiempo, como pueden ser los programas de ensayos de aptitud o proyectos repetidos como el EurA1c, pueden orientar sobre las causas de desempeños no satisfactorios, además, si estas causas son inherentes al desempeño del laboratorio o del instrumento empleado. El modelo de la TF-HbA1c es adecuado para evaluar el estatus de calidad en diferentes niveles y definir los objetivos de calidad en cualquiera de éstos debido a su flexibilidad, pero además puede ser un apoyo en la selección del instrumento para las determinaciones de $\mathrm{HbA} 1 \mathrm{c}$, considerando que debe ser elegido mediante un estricto proceso que incluya la revisión de los reportes de desempeño disponibles, como el EurA1c, para asegurarse de que cumpla con la calidad requerida para el uso clínico previsto, ${ }^{22}$ teniendo en cuenta que el objetivo de calidad debe ser más riguroso si se emplea en diagnóstico de diabetes. ${ }^{23}$

Existen diferencias del desempeño entre países que pueden deberse a múltiples factores tales como económicos, diferencias en los requerimientos o la falta de éstos, baja motivación o entrenamiento, uso de sistemas heterogéneos o hasta el cambio continuo de lotes y calibradores. Un factor adicional y que resulta primordial considerar es la diferencia de equipamiento entre países, por ejemplo, el grupo mexicano en el EurA1c emplea en un mayor porcentaje de equipos basados en inmunoensayo (51\%) y HPLC en casi la mitad de éstos (27\%). En cambio, los países europeos participantes utilizan mayoritariamente HPLC (55\%). ${ }^{8}$ Esto resulta determinante si se considera que la calidad de un ensayo para $\mathrm{HbA1c}$ está estrechamente ligada al equipo que se utiliza. ${ }^{24}$

El objetivo de calidad propuesto por la TF-HbA1c en las determinaciones de $\mathrm{HbA} 1 \mathrm{c}$ fue establecido como un punto de partida y se propuso su periódica revisión. Un destacado reporte señala que los instrumentos de uso rutinario tienen la capacidad para cumplir con objetivos más rigurosos, como reducir el TAE a $6 \%$ y un valor de $2 \sigma$, pero también sugiere que por ahora el objetivo sea lograr que $100 \%$ de los instrumentos cumplan el objetivo de calidad básico para después hacerlo más estricto, ${ }^{25}$ postura que compartimos, pues como se muestra aquí varios países que participan en este tipo de programas aún no consiguen este nivel de rendimiento y sólo se puede especular qué nivel tendrán países que no realizan ningún tipo de evaluación.

En resumen, con la aplicación del modelo para objetivo de calidad de la TF-HbA1c, es posible llevar a cabo una evaluación del estatus de calidad en los 
diferentes niveles aquí considerados; sin embargo, esta evaluación sólo puede ser realizada de forma concluyente cuando se utilizan materiales de primer nivel como los enunciados en este trabajo. La participación de este grupo de laboratorios mexicanos en proyectos internacionales permite estimar un «estado del arte» en nuestro país como se ha realizado en otros países, ${ }^{26}$ siendo este el segundo reporte que generamos sobre el desempeño en laboratorios mexicanos usando muestras con valor asignado por el MRP, y aunque los participantes y equipos son diferentes, es posible considerar que el desempeño en general ha mejorado, así como el equipamiento utilizado. ${ }^{27}$

\section{CONCLUSIONES}

Esperamos que los resultados aquí descritos sean un estímulo para continuar y aumentar la participación en estos importantes proyectos internacionales, esto es importante debido a la mejora que esto conlleva, como lo demostramos aquí. Además, alcanzar niveles óptimos de desempeño tiene un gran impacto en la calidad de la atención que reciben los pacientes con diabetes. La consecución de este tipo de metas requiere tiempo, siendo primordial la participación coordinada de todas las partes implicadas.

\section{AGRADECIMIENTOS}

Deseamos expresar nuestro agradecimiento a MSc. Carla Siebelder y al personal del Laboratorio de Referencia Europeo en Glicohemoglobina y del Laboratorio MCA Koningin Beatrix en los Países Bajos, por los datos proporcionados y su colaboración para este artículo.

A todos los laboratorios mexicanos participantes en el EurA1c en sus distintas ediciones.

A la Ing. Matsuri Rojano-Nisimura (Candidato PhD) por su apoyo en la revisión de este artículo.

\section{REFERENCIAS}

1. Diabetes Control and Complications Trial Research Group, Nathan DM, Genuth S, Lachin J, Cleary P, Crofford O et al. The effect of intensive treatment of diabetes on the development and progression of long-term complications in insulin-dependent diabetes mellitus. N Engl J Med. 1993; 329 (14): 977-986.

2. Turner R, Cull C, Holman R. United Kingdom Prospective Diabetes Study 17: a 9-year update of a randomized, controlled trial on the effect of improved metabolic control on complications in non-insulin-dependent diabetes mellitus. Ann Intern Med. 1996; 124 (1 Pt 2): 136-145.

3. Hoelzel W, Miedema K. Development of a reference system for the international standardization of $\mathrm{HbA} 1 \mathrm{c} / g l y c o h e m o g l o b i n$ determinations. J Int Fed Clin Chem. 1996; 8 (2): 62-64, 66-67.
4. Finke A, Kobold U, Hoelzel W, Weykamp C, Miedema K, Jeppsson JO. Preparation of a candidate primary reference material for the international standardisation of $\mathrm{HbA1c}$ determinations. Clin Chem Lab Med. 1998; 36 (5): 299-308.

5. Weykamp CW, Penders TJ, Muskiet FA, van der Slik W. Evaluation of a reference material for glycated haemoglobin. Eur J Clin Chem Clin Biochem. 1996; 34 (1): 67-72.

6. Jeppsson JO, Kobold U, Barr J, Finke A, Hoelzel W, Hoshino T et al. Approved IFCC reference method for the measurement of HbA1c in human blood. Clin Chem Lab Med. 2002; 40 (1): 78-89.

7. Hanas R, John G; International HBA1c Consensus Committee. 2010 consensus statement on the worldwide standardization of the hemoglobin A1C measurement. Diabetes Care. 2010; 33 (8): 1903-1904.

8. NGSP/IFCC 2019 Manufacturers World Forum. [Accesado junio 2020] Available in: http://www.ngsp.org/docs/MF2019.pdf

9. Task Force on Implementation of HbA1c Standardization (Integrated Project) (TF-HbA1c). [Accesado mayo 2020] Available in: https:// www.ifcc.org/executive-board-and-council/eb-task-forces/closedtask-forces/task-force-hba1c/

10. Weykamp C, John G, Gillery P, English E, Ji L, Lenters-Westra E et al. Investigation of 2 models to set and evaluate quality targets for hb a1c: biological variation and sigma-metrics. Clin Chem. 2015; 61 (5): 752-759.

11. A new IFCC Committee on Education in the Use of Biomarkers in Diabetes. Available in: http://www.ifcc.org/ifcc-news/archive2016/2016-01-14-c-eubd

12. The European Reference Laboratory for Glycohemoglobin. [Accesado junio 2020] Available in: www.euroreflab.com

13. EurA1c Trial Group. EurA1c: The European HbA1c trial to investigate the performance of HbA1c assays in 2166 laboratories across 17 countries and 24 manufacturers by use of the IFCC model for quality targets. Clin Chem. 2018; 64 (8): 1183-1192.

14. Westgard JO. Internal quality control: planning and implementation strategies. Ann Clin Biochem. 2003; 40 (Pt 6): 593-611. doi: 10.1258/000456303770367199.

15. Kallner A, McQueen M, Heuck C. The Stockholm Consensus Conference on quality specifications in laboratory medicine, 25-26 April 1999. Scand J Clin Lab Invest. 1999; 59 (7): 475-476.

16. American Diabetes Association. 2. Classification and diagnosis of diabetes: standards of medical care in diabetes-2020. Diabetes Care. 2020; 43 (Suppl 1): S14-S31.

17. Little RR, Rohlfing C, Sacks DB. The National glycohemoglobin standardization program: over 20 years of improving hemoglobin A1c measurement. Clin Chem. 2019; 65 (7): 839-848.

18. College of American Pathologists (CAP) GH5 Survey Data: (updated 12/19). [Accesado junio 2020] Available in: http://ngsp.org/CAP/ CAP19c.pdf

19. College of American Pathologists (CAP) GH5 Survey Data: (updated 6/19). [Accesado junio 2020] Available in: www.http://ngsp.org/ CAP/CAP19a.pdf

20. Weykamp CW, Mosca A, Gillery P, Panteghini M. The analytical goals for hemoglobin A(1 c) measurement in IFCC units and National Glycohemoglobin Standardization Program Units are different. Clin Chem. 2011; 57 (8): 1204-1246.

21. Kilpatrick ES. Toward a global overview of $\mathrm{HbA} 1 \mathrm{c}$ test performance. Clin Chem. 2018; 64 (8): 1131-1132.

22. Westgard JF. Quality requirements and standards. [Accesado mayo 2020] Available in: https://www.westgard.com/hba1c-2014partthree.htm

23. Heinemann L, Kaiser P, Freckmann G, Grote-Koska D, Kerner W, Landgraf R et al. Higher HbA1c measurement quality standards are 
needed for follow-up and diagnosis: experience and analyses from germany. Horm Metab Res. 2018; 50 (10): 728-734.

24. Kaiser P, Spannagl M, van Campenhout C, Lenga Y, Siebelder C, Weykamp C. HbA1c: EQA in Germany, Belgium and the Netherlands using fresh whole blood samples with target values assigned with the IFCC reference system. Clin Chem Lab Med. 2016; 54 (11): 1769-1775.

25. Lenters-Westra E, English E. Understanding the use of sigma metrics in hemoglobin A1c analysis. Clin Lab Med. 2017; 37 (1): 57-71.
26. Mosca A, Paleari R, Carobene A, Weykamp C, Ceriotti F. Performance of glycated hemoglobin $(\mathrm{HbA}(1 \mathrm{c}))$ methods evaluated with EQAS studies using fresh blood samples: still space for improvements. Clin Chim Acta. 2015; 451 (Pt B): 305-309.

27. Rojano-Rodríguez E, Acosta-González RI, Bocanegra-Alonso A, Rivera-Sánchez G, Sierra-Amor RI. Desempeño de un grupo de laboratorios mexicanos en la determinación de HbA1c. Bioquimia. 2007; 32 (3): 91-99. 